\title{
Accurate Microwave Technique of Surface Resistance Measurement of Large-Area HTS Films Using Sapphire Quasi-Optical Resonator
}

\author{
Nickolay Cherpak, Senior Member, IEEE, Alexander Barannik, Yury Filipov, Yury Prokopenko, and \\ Svetlana Vitusevich
}

\begin{abstract}
We have developed a surface resistance $\left(R_{s}\right)$ measurement technique for large-area high-temperature superconducting (HTS) films using quasioptical dielectric resonators (QDR) with HTS endplates (quasioptical Hakki-Coleman resonators). In this technique, the highest $Q$ modes, namely whispering-gallery modes, in sapphire disk sandwiched between HTS films or between one HTS film and one Cu endplate are excited at K-band frequencies. The authors report on measurement results of surface resistance of $52 \mathrm{~mm}$ diameter high-quality YBCO thin films. The measurement results revealed that the technique is feasible for accurate $R_{s}$-measurements of large-area thin films. The method is appropriate for standard measurement of $R_{s}$ at millimeter wave frequencies by analogy with classic DR-based microwave technique, although QDR-based technique has some fundamental differences.
\end{abstract}

Index Terms-Films, millimeter wave measurements, resonator, superconductors (high-temperature).

\section{INTRODUCTION}

A MONG a large variety of the resonant measurement systems, special emphasis is made on resonators developed for surface resistance, $R_{s}$, characterization of HTS unpatterned films [1]. The $R_{s}$-measurements are important both for fundamental physical studies and technical applications. The $R_{s}$-values of HTS films are sensitive to any kind of deffects in superconductors and to fabrication processes. By now, a number of groups have developed different techniques for the HTS film $R_{s}$-measurement [2].

The accuracy and sensitivity of the HTS film $R_{s}$-measurement depend on relation between losses caused by the resonator different parts. Physical studies and engineering developments using HTS materials call for standard techniques of $R_{s}$-measurement which should ensure high sensitivity, low error of measurements and relative ease of a measurement process [3].

For the classical microwave band (with frequencies $f$ less than $25 \mathrm{GHz}$,) the standard technique based on a sapphire

Manuscript received August 6, 2002. This work relates to Department of the Navy Grant N00014-02-1-4072 issued by the Office of Naval Research International Field Office. The United States Government has a royalty-free license throughout the world in all copyrightable material contained herein.

N. Cherpak, A. Barannik, Y. Filipov, and Y. Prokopenko are with Usikov Institute of Radiophysics and Electronics, National Academy of Sciences, Kharkiv, 61085, Ukraine (e-mail: cherpak@ire.kharkov.ua; prokopen@ire.kharkov.ua).

S. Vitusevich is with the Institut für Schichten und Grenzflächen Forschungszentrum, Jülich, D-52425, Germany (e-mail: s.vitusevich@ fz.-juelich.de).

Digital Object Identifier 10.1109/TASC.2003.812400
$\left(\mathrm{Al}_{2} \mathrm{O}_{3}\right)$ resonator with the lower mode oscillations has been proposed [4]. Of two basic types of a dielectric resonator (DR), a Hakki-Coleman resonator [5]. has the advantage of permitting the calculation of the total energy dissipation.

In the millimeter $(\mathrm{mm})$ waverange ( $f$ is higher than $25 \mathrm{GHz}$ ), the dimensions of DR's with the lower-mode oscillations (waves) become unacceptably small and their quality factor, $Q$, falls. The highest azimuthal order modes, i.e., whispering gallery (WG) waves, have the highest $Q$-quality factor. The devices are quasioptical, so DR's with two conducting endplates (CEP) and WG waves are, as a matter of fact, the quasioptical (QDR) Hakki-Coleman resonators [6]. They have acceptable dimensions in $\mathrm{mm}$ waverange and are promising for using in resonant structures with HTS films.

A consistent analysis of electrodynamic properties of the QDR with CEP in a case of anisotropic dielectric [7] provides theoretical foundation for the QDR-based $R_{S}$ determination. The present work deals with the following problems: i) quantitative evaluation of radiation, dielectric and (super)conductor energy losses in QDR with CEP; ii) experimental justification of validity of the proposed $R_{s}$-measurement technique; iii) $R_{s}$-measurement of HTS large-area films in the mm wave range using QDR with CEP.

\section{THEORETICAL RELATIONSHIPS FOR THE $Q$-FACTOR OF THE QUASI-OPTICAL DIELECTRIC RESONATOR WITH CONDUCTING ENDPLATES}

In accordance with a sum rule for inverse $Q$-values, the expression for eigen $Q$-factor of QDR with CEP can be written as

$$
Q_{0}^{-1}=k \tan \delta+\frac{1}{2} A_{s}\left(R_{s}+R_{s N}\right)+Q_{\text {rad }}^{-1}
$$

where the coefficients $k$ and $A_{s}$ show contribution of dielectric and conductor losses in the energy total loss in QDR, $\tan \delta$ is the dielectric loss tangent, $Q_{\text {rad }}$ is the radiation loss quality, $R_{s}$ and $R_{S N}$ are the surface resistances of the HTS film and normal metal CEP's. As is shown below, one can neglect $Q_{\text {rad }}^{-1}$ in the QDR with CEP with large diameter. In a case when both CEP's are formed of HTS samples (normal metal), we obtain

$$
Q_{0}^{-1}=k \tan \delta+A_{s} R_{s(N)} .
$$

From the electrodynamic solution of the field structure in the QDR with CEP, the expression for $Q$-quality is obtained [7]

$$
Q_{0}^{-1}=\frac{1}{1+R_{0}^{Y}} \tan \delta+\frac{2}{\omega \mu_{0} l R_{Y}} R_{s},
$$


where " $Y$ " denotes the wave mode, $H E_{n s m}$ or $E H_{n s m}$, and $n$, $s, m$ are the azimuthal, radial and axial numbers, respectively. From (2) and (3), it follows that

$$
k=\frac{1}{1\left(1+R_{0}^{Y}\right)}, A_{s}=\frac{2}{2 \omega \mu_{0} l R^{Y}},
$$

where $l$ is the height of a dielectric cylinder and $\mu_{0}=4 \pi \times$ $10^{-7} \mathrm{H} / \mathrm{m}$. The expressions for $R_{0}^{Y}$ and $R_{Y}$ depend on dimensions of the resonator and the wave mode within it. Here they are not cited because they are extremely clumsy. However in the case of axially homogeneous $H E_{n s 0^{-}}$mode which is excited relatively easy, the expressions for $R_{H E}=R_{Y}$ and $R_{0}^{H E}=R_{0}^{Y}$ in (3)-(4) are simplified considerably (because the wave number $k_{z}=0$ )

$$
\begin{aligned}
& R_{H E}= \\
& \frac{\varepsilon_{\|}\left[F_{1}\left(q_{E} a\right)+\frac{1}{2} F_{2}\left(q_{E} a\right)\right]-\frac{\left|J_{n}\left(q_{E} a\right)\right|^{2}}{\left|H_{n}^{(1)}\left(q_{0} a\right)\right|^{2}} \cdot\left[\Phi_{1}\left(q_{0} a\right)+\frac{1}{2} \Phi_{2}\left(q_{0} a\right)\right]}{\varepsilon_{\|}\left[F_{1}\left(q_{E} a\right)+F_{2}\left(q_{E} a\right)\right]-\frac{\left|J_{n}\left(q_{E} a\right)\right|^{2}}{\left|H_{n}^{(1)}\left(q_{0} a\right)\right|^{2}} \cdot\left[\Phi_{1}\left(q_{0} a\right)+\Phi_{2}\left(q_{0} a\right)\right]},
\end{aligned}
$$$$
R_{0}^{H E}=\frac{\frac{\left|J_{n}\left(q_{E} a\right)\right|^{2}}{\left|H_{n}^{(1)}\left(q_{0} a\right)\right|^{2}}\left[\Phi_{1}\left(q_{0} a\right)+\frac{1}{2} \Phi_{2}\left(q_{0} a\right)\right]}{\varepsilon_{||}\left[F_{1}\left(q_{E} a\right)+\frac{1}{2} F_{2}\left(q_{E} a\right)\right]}
$$

where

$$
\begin{aligned}
& F_{1}\left(q_{E} a\right)=\left(1-\frac{n^{2}}{q_{E}^{2} a^{2}}\right) J_{n}^{2}\left(q_{E} a\right)+J_{n}^{\prime 2}\left(q_{E} a\right) \\
& F_{2}\left(q_{E} a\right)=\frac{1}{q_{E} a}\left[J_{n}\left(q_{E} a\right) J_{n}^{\prime *}\left(q_{E} a\right)+J_{n}^{*}\left(q_{E} a\right) J_{n}^{\prime}\left(q_{E} a\right)\right] .
\end{aligned}
$$

Similar to the expressions for $F_{1(2)}\left(q_{E} a\right)$, the corresponding relations for $\Phi_{1(2)}\left(q_{0} a\right)$ in terms of the $q_{0} a$ and $H_{n}^{(1)}\left(q_{0} a\right)$ functions take place. Here $q_{0}=k_{0}=\omega / c ; q_{E}^{2}=\varepsilon_{\|} k_{0}^{2} ; a$ is the dielectric disk radius, $J_{n}(x)$ and $H_{n}^{(1)}(x)$ are the $n$-th order Bessel and Hankel cylindrical functions of the first kind; $n$ is integer, prime stands for derivative, $\varepsilon_{\|}$is the dielectric permittivity component in the direction parallel to the crystal optic axis.

Defining $Q_{\text {rad }}=\omega^{\prime} / 2 \omega^{\prime \prime}$, where the complex frequency $\omega=$ $\omega^{\prime}-i \omega^{\prime \prime}$ is found from the dispersion equation [7], we obtain $Q_{r a d}$-value which is more than $10^{9}-10^{10}$. Then one can really neglect the $Q_{\text {rad }}^{-1}$ term in (1).

\section{EXPERIMENTAL DETAILS}

The measurements were carried out in the frequency band from 35 to $37 \mathrm{GHz}$. For the experimental studies, a measuring device was made (Fig. 1), the main element of which is a sapphire cylindrical disk $d=2 a=14 \mathrm{~mm}$ in diameter and $l=$ $2.4 \mathrm{~mm}$ in height. The optical $c$-axis of the sapphire was directed at the angle 56 with respect to the longitudinal one. A single crystal sapphire has been synthesized by the directed crystallization technique.

Free-oxygen copper, unannealed and annealed, and high- $T_{c}$ superconducting $\mathrm{YBa}_{2} \mathrm{Cu}_{3} \mathrm{O}_{7-\delta}$ thin films served as the CEP's. The HTS films were deposited onto single crystal sapphire substrates of $52 \mathrm{~mm}$ in diameter using the laser ablation technique. The film thickness is $330 \mathrm{~nm}$ ( $\pm 10 \%$ or less). The film thickness

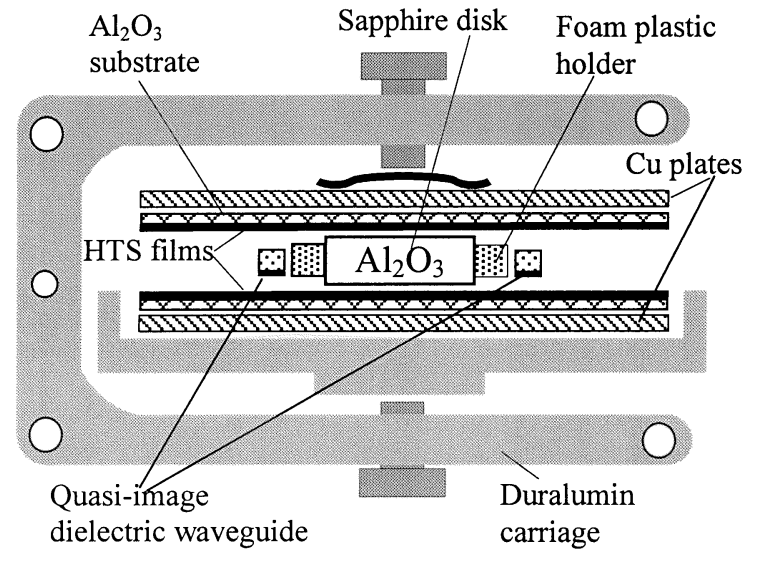

Fig. 1. Schematic representation of a unit with sapphire QDR for surface resistance measurements of HTS films.

TABLE I

Calculated $k, A_{s}$ AND $Q_{r a d}$-Factor Values

\begin{tabular}{llccc}
\hline \hline $\begin{array}{c}\text { Temperature } \\
T, \mathrm{~K}\end{array}$ & $\begin{array}{c}\text { Frequency } \\
f, \mathrm{GHz}\end{array}$ & $\begin{array}{c}\text { Coefficient } \\
k\end{array}$ & $\begin{array}{c}\text { Coefficient } \\
A_{s}, 1 / \mathrm{m} \Omega\end{array}$ & $\begin{array}{c}\text { Radiation } \\
\text { quality } \\
Q_{\text {rad }}\end{array}$ \\
\hline 77 & 35.57 & 0.992 & $2.924 \cdot 10^{-6}$ & $5.3 \cdot 10^{9}$ \\
300 & 35.12 & 0.992 & $2.961 \cdot 10^{-6}$ & $5.3 \cdot 10^{9}$ \\
\hline \hline
\end{tabular}

scatter within the substrate area is $<5 \%$. The critical characteristics are $T_{c} \geq 87 \mathrm{~K}$ and $J_{c} \geq 1.5 \cdot 10^{6} \mathrm{~A} / \mathrm{cm}^{2}$ at $77 \mathrm{~K}$. The density of defects is as follows: absence of scratches and linear defects and absence of striking particles or point defects with diameter larger then $25 \mu \mathrm{m}$.

The coupling of QDR with transmission lines is realized by means of dielectrics waveguides one side of which is covered by a metal. Input and output waveguides are directed at some angle $\left(\sim 10^{\circ}\right)$ with respect to each other. This allows one to control the coupling of the QDR with the feeder lines by moving the resonator along the lines. The coupling control can be carried out during the measurement process as the temperature changes. The approach makes it possible to extend a dynamic range of measured $R_{s}$-values. The feeder lines were also manufactured of the sapphire.

\section{A. Results and Discussion}

As follows from (1) and (2), for $R_{s}$-determination it is necessary to know $\tan \delta$ and $R_{S N}$ (in the case when only one HTS film is used and another CEP is a normal metal), to calculate $k$ and $A_{s}$ coefficients and to measure $Q$-factor. Here it is necessary also to identify the wave mode used for $R_{s}$-measurements. As mentioned above, the wave modes $H E_{n s 0}$ are excited quite easily. In the given frequency band we deal with the wave mode which is characterized by $n=14$ and $s=1$. All of the calculated $k, R_{H E}$ and $A_{s}$ coefficients and $Q_{r a d}$-factor used for determination of $R_{s}$ are given in Table I.

The single crystal sapphire has the lowest $\tan \delta$ among the known solid-state dielectrics (maybe except diamond). Up to now a sapphire has been studied quite enough (see for example [7], [8]). However the value of sapphire $\tan \delta$ depends strongly on crystal perfection. In addition, this value is determined by 


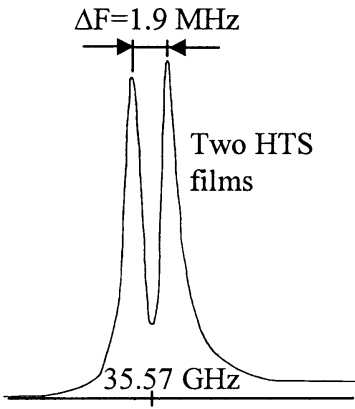

(a)

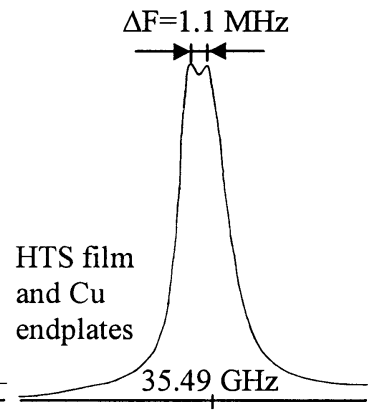

(b)
Fig. 2. Splitted resonant lines of QDR with (a) two HTS film endplates and (b) one HTS film and one $\mathrm{Cu}$ endplates.

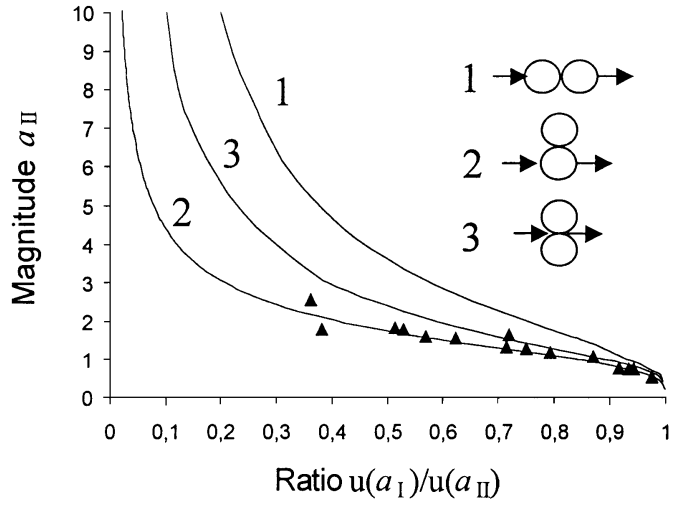

Fig. 3. Partial resonant frequency in units of frequency generalized detuning as a function of ratio of minimal amplitude to maximal one of the QDR amplitude-frequency response.

means of $Q$-measurement of the open QDR and $Q$-factor in turn depends on the resonator surface machining, a departure of the resonator form from the ideal round cylinder and accuracy of $c$-axis orientation. The above mentioned factors necessitate measuring of $\tan \delta$ in every certain case. In the present work, $\tan \delta$ is determined by means of $Q$-measurement of the open QDR $H E_{121} \delta$-mode. Measured values of $\tan \delta$ are equal to $3.1 \cdot 10^{-5}$ at $300 \mathrm{~K}$ and $2.9 \cdot 10^{-6}$ at $77 \mathrm{~K}, \log (\tan \delta)$ being properly described by a linear function of temperature, $T$ [8], [9].

At $R_{s}$-measurements of high-quality HTS films, forming CEP, one can observe a splitting of the resonant line, even in the case of one film (Fig. 2). The splitted resonance indicates the removal of degeneracy. Indeed, each of the higher order azimuthal waves is two-fold degenerate one. The equivalent circuit, describing the QDR with CEP in this case is presented by two oscillatory circuits with certain coupling between them. The approach developed in this work allows one to find the $Q$-factor of the single oscillatory circuit using three measured parameters, namely, resonant frequency $f_{0}$ of the single uncoupled circuit, the ratio between minimal and maximal amplitudes $u\left(a_{I}\right) / u\left(a_{I I}\right)$ of the resonator amplitude-frequency response and difference $\Delta F=f_{2}-f_{1}$ of coupled $f_{1}$ and $f_{2}$ frequencies of the QDR with the splitted resonant line. Here $a_{I}$ and $a_{I I}$ are frequency generalized detunings $a=\left(f-f_{0}\right) Q_{0} / f_{0}$ at $f=f_{0}$ and $f=f_{2}$ respectively, $f_{2}-f_{0}=\left|f_{1}-f_{0}\right|$. Fig. 3 shows that only one of three possible equivalent circuits (corresponding to
TABLE II

$R_{s}$-VALUe OF CU AND $\mathrm{YBa}_{2} \mathrm{Cu}_{3} \mathrm{O}_{7-\delta}$ FILMS IN $K_{a}$-BAND.

\begin{tabular}{lllll}
\hline \hline & $\begin{array}{c}\text { Temperature } \\
T, \mathrm{~K}\end{array}$ & $\begin{array}{c}\text { Quality } \\
\text { factor }\end{array}$ & $\begin{array}{c}R_{s} \\
(\text { measured }) \\
\mathrm{m} \Omega\end{array}$ & $\begin{array}{c}R_{s} \\
\text { (calculated) } \\
\mathrm{m} \Omega\end{array}$ \\
\hline $\begin{array}{l}\mathrm{Cu} \\
\text { unannealed }\end{array}$ & 300 & 5100 & 55.9 & 48.4 \\
$\mathrm{Cu}$ annealed & 300 & 5730 & 48.8 & 48.4 \\
$\mathrm{YBa}_{2} \mathrm{Cu}_{3} \mathrm{O}_{7-8}$ & 77 & 37170 & 8.2 & - \\
\hline \hline
\end{tabular}

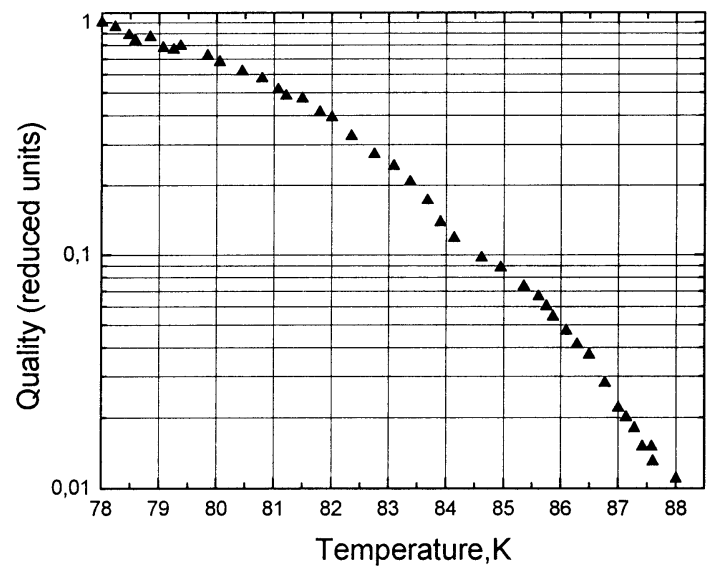

Fig. 4. Temperature dependence of $Q$-factor for QDR with one HTS film.

curve 2) describes the experimental data. From Fig. 3 we can find

$$
a_{I I}=\frac{\Delta F}{2 f_{0}} Q_{0} .
$$

Thus, we obtain a good chance to find from (7).

$R_{s}$-values of the oxygen-free copper and HTS film together with $Q$-values and corresponding frequencies of QDR are listed in Table II. It follows from Table II that measured $R_{S}$ of the annealed copper is in a good agreement with $R_{s}$ value calculated using the expression $R_{s}=\sqrt{\omega \mu_{0} / 2 \sigma}$ at $300 \mathrm{~K}$ when the normal skin-effect takes place, where $\sigma$ is conductivity. This result is an additional argument of the validity of the proposed approach to the analysis of field structure in the QDR with CEP and calculation of $k$ and $A_{s}$ coefficients based on the developed analysis.

Fig. 4 shows the temperature dependence of the signal proportional to $Q_{0}$, from which one can see that we have not limitation on $R_{s}$-measurement in the entire temperature range used. The measurement technique allows studying dependence of $R_{s}$ in broad dynamic range on such external factors as temperature, dc magnetic field and strong microwave fields. Study of HTS nonlinear properties using QDR with CEP has advantages, conditioned by presence of natural isolation of channels with different frequencies, which coincide with the QDR eigenfrequencies.

The most probable error of $R_{s}$-measurement is evaluated by the known expression [4] and is about 5\%. It can be further reduced by using an advanced measurement technique. 


\section{CONCLUSION}

Thus, justification of the proposed technique of HTS film $R_{s}$-measurement using quasioptical dielectric resonators with HTS endplates in the mm waveband is presented. Fundamental differences between the known DR-based technique and proposed QDR-based technique are as follows: i) dimensions of the QDR are quite acceptable in the millimeter waveband which allows one to perform experiments in a shorter wavelength range; ii) the resonance line is splitted in the case of any inhomogeneity in QDR with high quality factor. We have shown that even in the latter case it is possible to measure $Q$-value with a high degree of accuracy. In this case a new procedure of finding $Q$-factor is required. The proposed technique ensures the measurement high accuracy and can be considered as a standard technique for HTS large-area film $R_{s}$-measurement in the millimeter waveband.

\section{REFERENCES}

[1] G. Hein, High-Temperature-Superconductor Thin Films at Microwave Frequencies. Berlin, New York, Heidelberg: Springer, 1999, vol. 155, Springer Tracts in Modern Physics.
[2] M. L. Lancaster, Passive Microwave Devices Application of High Temperature Superconductors. Cambridge, UK: Cambridge Univ. Press, 1997.

[3] M. Misra, N. D. Kataria, R. Pinto, M. Tonouchi, and G. P. Srivastava, "Sensitivity of $R_{s}$-measurement of HTS thin films by three prime resonant techniques: Cavity resonator, dielectric resonator and microstrip resonator," IEEE Trans. Appl. Supercond., vol. 11, pp. 4140-4147, Dec. 2001

[4] J. Mazierska and C. Wilker, "Accuracy issues in surface resistance measurement of high temperature superconductors using dielectric resonators," IEEE Trans. Appl. Supercond., vol. 11, no. 1, pp. 3217-3225, 2001.

[5] B. W. Hakki and P. D. Coleman, "A dielectric resonator method of measuring inductive capacities in the millimeter range," IEEE Trans. Microwave Theory, vol. 8, pp. 402-410, 1960.

[6] N. T. Cherpak, "Mm wave resonant ant nonresonant structures with high- $T_{c}$ superconductors," in MSMW'2001 Symposium Proc., vol. 1, Kharkov, 2001, pp. 63-68.

[7] A. A. Barannik, Y. V. Prokopenko, Y. F. Filipov, and N. T. Cherpak, "Quasioptical dielectric resonator. Microwave characterization of dielectrics and conductors," Telecommunications and Radioengineering, vol. 55, no. 12, pp. 360-362, 2001.

[8] V. B. Braginsky, V. S. Il'chenko, and K. S. Bagdassarov, "Experimental observations of fundamental microwave absorption in highly-quality dielectric crystals," Phys. Lett. A, vol. 120, no. 6, pp. 300-305, 1987.

[9] E. M. Ganapolskii, "Absorption of microwave band electromagnetic field in perfect dielectric crystals," Low Temperature Physics, vol. 26 , no. 11, pp. 862-864, 2000. 